\title{
Transmitters for two-tier optical data-packet labelling in advanced IP networks
}

\author{
R. O'Dowd, Y. Yu, G. Mulvihill, S. O'Duill, G. Morthier and B. Moeyersoon
}

\begin{abstract}
Semiconductor lasers send information over the Internet in packets. Evaluation of a new transmitter concept for optical data packet switching suited to optical networks that can switch channels rapidly is presented. In such networks optical paths are established that are switched using labels and a wavelength label, in addition to a frequency-modulated tone as a second label, that is encoded by optical frequency modulation, FM or FSK, is used. The new second label allows for fast re-assignment of the packet to an alternative transport means in the fibre if required. Both simulation and experiments have been performed to assess transmitter performance of the proposed combined-labelling scheme. To this end, a widely tunable laser is used to convert the colour (wavelength) and thereby efficiently route the optically labelled packets in a network. At the receiver end the data can be re-assembled using the colour as a guide, even if it were sent in bursts. Within the EU-STOLAS project, laser performance simulations have been done and fast measurements carried out at specific ITU-channel wavelengths.
\end{abstract}

\section{Introduction}

Owing to the recent slow-down in the telecommunications industry, research and development teams worldwide are trying hard to develop the technology and the systems that will allow the industry to offer cost effective and reliable solutions to the end-user. Semiconductor lasers are used today to send information on the Internet at a suitable optical wavelength over optical fibre. The emergence of WDM technology (multiple wavelengths on each fibre) has unlocked most of the available bandwidth, leading to lower costs, which can be expected to further fuel the demand for broad bandwidth. However dense WDM does not hold all the answers. The increasing transmission rates will eventually shift the bottleneck from the transport cable back to the switching centres. Indeed, today's switching technologies as deployed are capable of switching at rates of 'only' up to $10 \mathrm{Gbit} / \mathrm{s}$. While emerging switches and Internet protocol (IP) routers can be used to switch data using the individual channels within a WDM link (the channels typically operate at $2.4 \mathrm{Gbit} / \mathrm{s}$ or $10 \mathrm{Gbit} / \mathrm{s}$ ), this approach implies that tens or hundreds of switch interfaces must be used to terminate a single cable link with a large number of wavelength channels. Moreover, there can be a significant loss of efficiency when the parallel channels are used simply as a collection of independent links, rather than as the new inter-working, shared resource demonstrated in the EU-STOLAS project. We achieve this channel interworking by optically labelling the data packets with a novel laser transmitter.

\section{(C) IEE, 2005}

IEE Proceedings online no. 20045010

doi: 10.1049/ip-opt:20045010

Paper first received 9th June 2004 and in revised form 13th April 2005 R. O'Dowd, Y. Yu, G. Mulvihill and S. O'Duill are with Dept. Electronic Engineering, University College Dublin, Ireland

G. Morthier and B. Moeyersoon are with IMEC University Gent Belgium E-mail: Ronan.ODowd@ucd.ie
The tunable laser in Fig. 1 is wavelength-controlled by two or three electronic currents. For the implementation of a tunable FM transmitter with a small optical frequency modulation, while maintaining a large signal integrity, modulation of the laser phase section current alone achieves a frequency 'dither' of $20 \mathrm{GHz}$ about the central wavelength and this would be adequate in order to separate and detect two optical tones at the receiver, assuming a normal WDM wavelength channel spacing of $50 \mathrm{GHz}$ optical. Low values of FM currents at certain wavelengths, however, prohibit modulation. This can be overcome by altering the dual tuning-current combination required for wavelength selection in the laser. It has been found that adjustment of the second current to higher values also requires higher FM currents for obtaining 32 fibre channels.

A new, optically tunable FM transmitter was implemented using a sophisticated, widely tunable laser. With the right control current settings, FM tones for 32 WDM channels can be achieved on a single optical fibre. The source can be used for optical labelling of IP data packets in a novel, packetswitched network or even where the data is sent in bursts to be re-assembled at the user's end. The laser-generated labels facilitate this re-assembly while improving routing options.

\section{Two-tier optical labelling specifications}

Tunable laser diodes play an important role in the intensity modulation/frequency shift keying (IM/FSK) optical labelling scheme [1]. In the scheme, the tunable laser rapidly switches between wavelength channels, to set up light paths through the nodes, and simultaneously generates an optical signal to provide another FSK label that identifies the label-switched path. Furthermore, within the EU-STOLAS project, the following specifications were determined according to network/system requirements [2]:

- Operating wavelength channels: on $200 \mathrm{GHz}$ ITU grids (192.1, 192.3, 192.5 and $192.7 \mathrm{THz})$, with an accuracy of $\pm 5 \mathrm{GHz}$.

- FSK frequency deviation: $\pm 10 \mathrm{GHz}(20 \mathrm{GHz}$ spacing from FSK tone to tone) around ITU grid channel. 


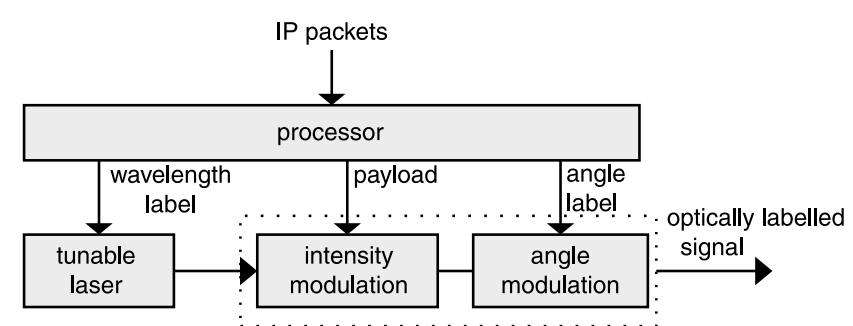

Fig. 1 Internet protocol or IP data packet entering the transmitter and being processed to convert it to a laser light output on an optical fibre

Wavelength is used to tag it to its destination while a second FSK label is also stamped on the packet; this allows the network to select the best type of connection from server to user and re-assemble at the receiver

- Maximum label rate for (burst/packet) header: 78, 125, $155 \mathrm{Mbit} / \mathrm{s}$.

- Minimum guard-band (minimum silent period between consecutive packets): $0.5-1.0 \mu \mathrm{s}$.

For this purpose, this paper will report our results on FSK modulation and wavelength switching properties of tunable lasers and compatibility with the network/system requirements.

In Section 3, implementation and performance of FSK modulation with the devices are described, involving foursection grating/co-directional coupler/sampled grating reflector (GCSR) lasers and a commercial three-section distributed Bragg reflector (DBR) laser. The former covers full C-band and the latter a quarter of this span. Static characterisations of the devices are first investigated in order to obtain indications of the FSK implementation scheme. Some important static performances are identified and discussed. Dynamic measurements are then implemented to explore FSK modulation capability of the devices. Importantly, FSK modulation at reasonable label information rates (78 Mbit/s) with $\pm 10 \mathrm{GHz}$ tones by using a single, phase section only is demonstrated.

In Section 4, FSK modulation behaviour of the devices is studied theoretically, numerically and experimentally where the reflector grating is modulated instead of the phase current. FSK modulation response and $3 \mathrm{~dB}$ cut-off frequency are analysed and compared for different bias currents. Results show that FSK modulation with threesection DBR lasers at $155 \mathrm{Mbit} / \mathrm{s}$ is feasible.

In Section 5, thermal effects on wavelength switching dynamics are investigated. Combining the carrier and thermal effects, full frequency modulation response of a GSCR laser is simulated and is compared to measurements. Degradation to wavelength switching time and control precision are demonstrated by dynamic measurements. Results indicate that a guard time of at least $500 \mathrm{~ns}$ is needed to minimise thermal effects so that the laser can switch from one wavelength to another within the accuracy of $\pm 5 \mathrm{GHz}$ as required by the passive demodulation optics, a linear-slope filter.

\section{Performance of C-band and quarter C-band lasers in FSK modulation}

In this Section, we will report results on FSK implementation and performance with various tunable lasers using the phase section for the second label. Each laser has its own look-up table for section currents that optimally output the required ITU wavelength, SMSR and power [3].

To obtain indications of FSK implementation capability, static characterisations are investigated first. Bias control, frequency deviation, modulation efficiency and power difference between the two FSK tones are identified. Dynamic measurements are then implemented to explore FSK modulation performance. Importantly, we show that FSK modulation at reasonable rates $(78-155 \mathrm{Mbit} / \mathrm{s})$ with $\pm 10 \mathrm{GHz}$ tones can be achieved by using the phase section only. The earlier concern that complicated ITU look-up table re-configuration for some channels might be required was eliminated.

Investigations were mainly carried out on two types of tunable laser diodes, i.e. a four-section GCSR laser and a three-section DBR laser. The latter requires a set of four devices to cover full $\mathrm{C}$-band

\subsection{Static characterisation and FSK implementation}

3.1.1 GCSR laser results: Static characterisation results of GCSR lasers have been presented in previous publications [4-6]. These static characterisation results provide valuable insight into the operation of the device and indicate limitations on the implementation of the FSK modulation scheme with the device. Frequency deviation in addition to modulation efficiency have been identified. It has been shown that it is possible to achieve FSK modulation with $\pm 10 \mathrm{GHz}$ tones by using phase section only.

3.1.2 DBR laser results: Static characterisation results of DBR lasers are shown in Figs. 2 and 3. The firs gives an example of optical frequency detailing behaviour at ITU channel $192.1 \mathrm{THz}$. Clearly, the operating point (marked with a dot) is well located within the safe operating region that avoids mode hopping. For a fixed reflector current, the performance of frequency control with phase current at the same channel is displayed in Fig. 2. It shows the typical quasi-periodic trend of frequency against phase current for DBR lasers, where there is a continuous tuning with a wavelength shift (frequency deviation) equal to the longitudinal mode spacing (about $50 \mathrm{GHz}$ ). As shown in Fig. 2, operating bias point is marked with a dot, where left frequency deviation is $17 \mathrm{GHz}$ and right frequency deviation is $35 \mathrm{GHz}$. This allows FSK implementation with phase section only to achieve $\pm 10 \mathrm{GHz}$ tones around the central channel frequency. Once the laser is biased at the above operating point, the phase current can be dithered to generate the two FSK tones.

Frequency deviation for all available 16 ITU channels has been summarised in Fig. 4. Left frequency deviation distributes $12-19 \mathrm{GHz}$ while right frequency distributes 31-40 GHz. That means, for all channels, FSK implementation with $\pm 10 \mathrm{GHz}$ tones by using phase section only can

frequency, $\mathrm{THz}$

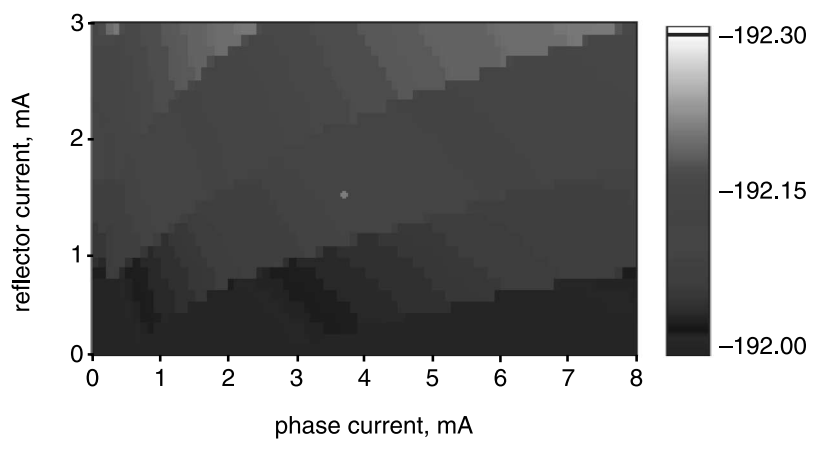

Fig. 2 Reflector current against phase current plane with scale for optical frequency detailing behaviour at ITU channel 192.1 THz for the DBR laser 


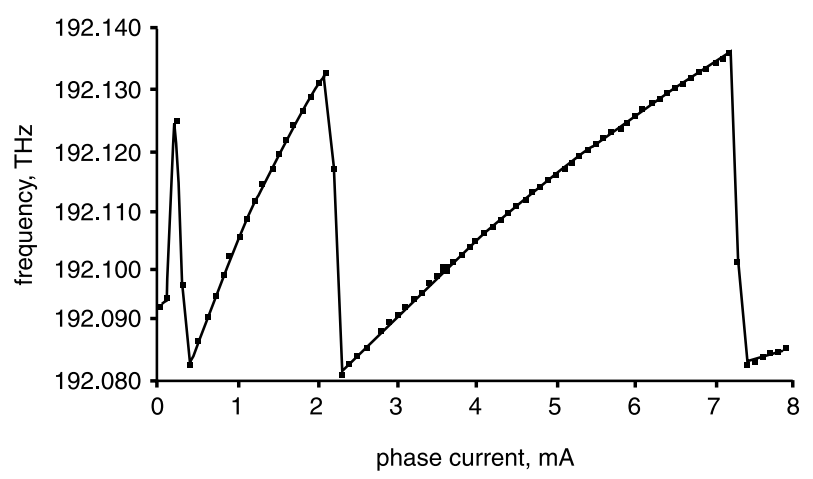

Fig. 3 Plot of frequency against phase current at ITU channel $192.1 \mathrm{THz}$ for the DBR laser

be achieved without modification to the bias current selections from the normal ITU look-up table.

Modulation efficiency is an important parameter for FSK transmitter design. From Fig. 3, it can be found that DC modulation efficiency is about $12.5 \mathrm{GHz} / \mathrm{mA}$ for the ITU $192.1 \mathrm{THz}$ channel. For all 16 channels, DC modulation efficiency varies between $11 \mathrm{GHz} / \mathrm{mA}$ to $22 \mathrm{GHz} / \mathrm{mA}$, depending on bias of phase current. This divergence of modulation efficiency among channels will add to drive complexity since modulation amplitude needs to be modified when the laser switches from one channel to another.

Power difference between the two FSK tones with IM/FSK signal transmission is also a concern. It is desirable to keep power variation between the two tones as low as possible to minimise residual intensity modulation since it may interfere with the intensity-modulated payload data and thus will cause a sensitivity penalty [7]. For the tunable lasers, current injection into tuning sections will cause a slight output power degradation [3,6]. For the GCSR laser to achieve optical frequency deviation of $20 \mathrm{GHz}$, the phase section modulation gives a $10 \%$ intensity fluctuation [6]. By contrast, to achieve the same frequency deviation, only $3 \%$ intensity variation is found for the DBR laser.

\subsection{Dynamic measurements and FSK modulation}

3.2.1 GCSR laser results: Previous dynamic measurement results of FSK modulation with the GCSR laser have been reported [6]. Time-averaged spectra of FSK modulation at low modulation rates were observed and measured with an optical spectrum analyser (OSA). It has been shown that two optical side tones at $\pm 10 \mathrm{GHz}$ around the central channel frequency can be achieved with the phase section modulation.

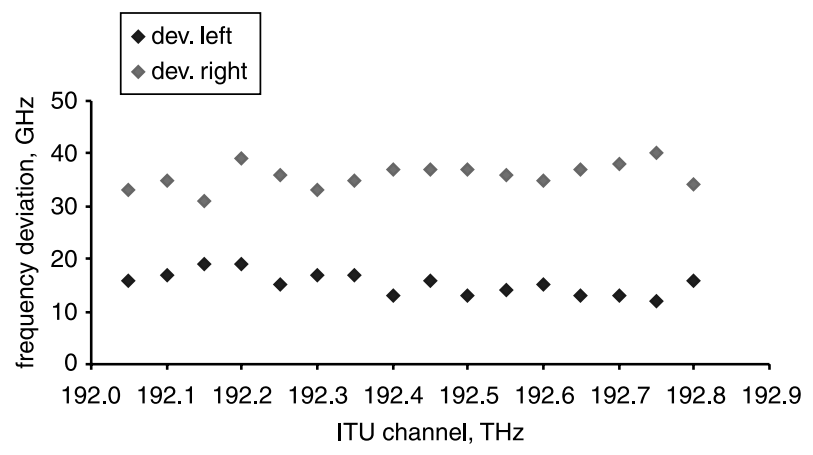

Fig. 4 Frequency deviation for 16 ITU channels of the DBR laser

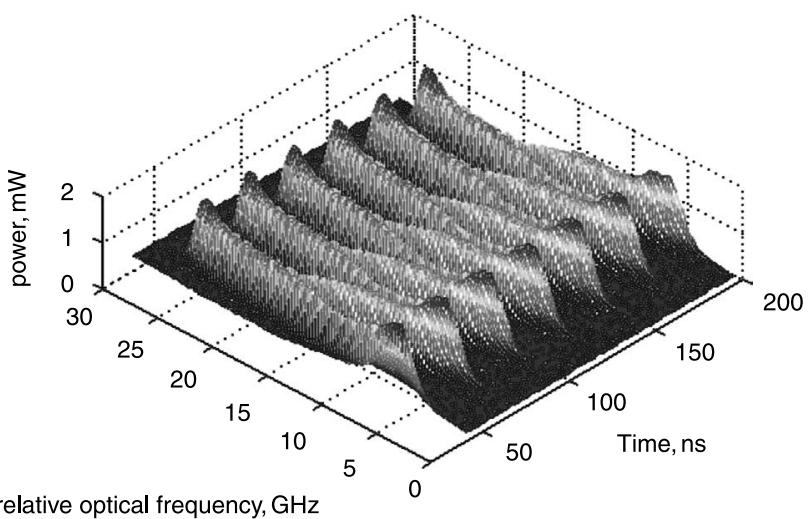

Fig. 5 Time-resolved spectrum of FSK modulation with the GCSR laser around ITU channel 195.2 THz

Further explorations were done with a time-resolved spectral (TRS) measurement technique based on a scanning Fabry-Perot interferometer (FPI) [4, 6]. This technique has been demonstrated to be very useful to characterise dynamics of the tunable lasers as it can measure optical signals with time-dependent spectra, where the frequency window can be selected for different applications. Figure 5 gives an example of measured results, showing the timeresolved spectrum of FSK modulation for the sample ITU channel at 195.2 THz. The laser spectrum switches between the two tones with time as can be clearly seen. As shown in Fig. 5, FSK deviation of $20 \mathrm{GHz}$ has been achieved with a modulation current of $3 \mathrm{~mA}$. Here the FSK modulation rate is $39 \mathrm{MHz}$, which corresponds to $78 \mathrm{Mbit} / \mathrm{s}$ FSK labelling rate in a non-return-to-zero (NRZ) coding scheme.

Figure 6 shows the frequency modulation (FM) response for the phase current at two different bias levels $(1.90 \mathrm{~mA}$ and $3.25 \mathrm{~mA}$ ). The FM efficiency is plotted against modulation frequency from 1 to $250 \mathrm{MHz}$. As shown in Fig. 6 , the $3 \mathrm{~dB}$ cutoff frequency is limited to about $50 \mathrm{MHz}$, which is ultimately determined by the carrier lifetime in the phase section in addition to the parasitic capacitance of the device. It is desirable to have higher FM bandwidth for higher FSK labelling bit-rate. Improvements on FM bandwidth can be achieved by optimisation of device design and impedance-matching circuits. However, the above limited modulation bandwidth can be tolerable for a relatively low bite-rate of header transmission, for example of $78 \mathrm{Mbit} / \mathrm{s}$.

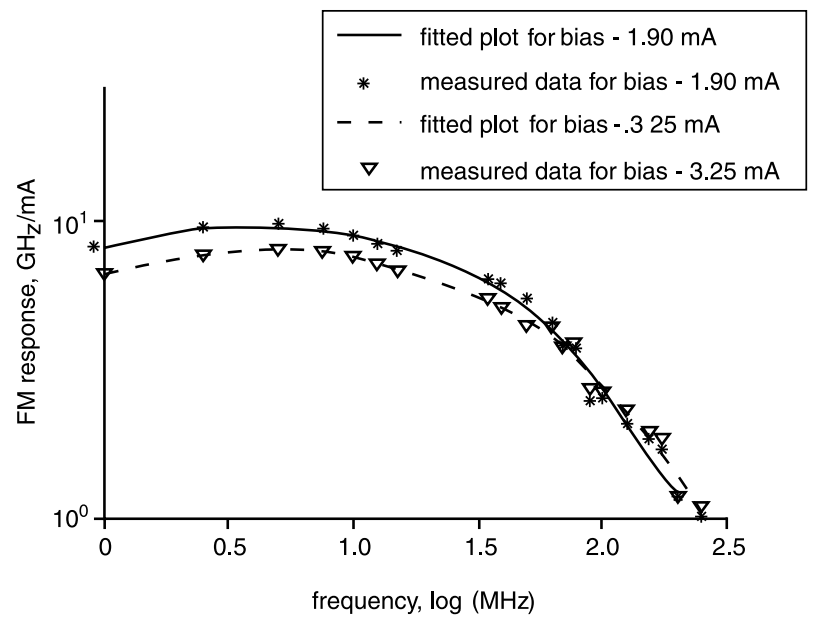

Fig. 6 FM response of the GCSR laser with phase section current in two different bias cases 


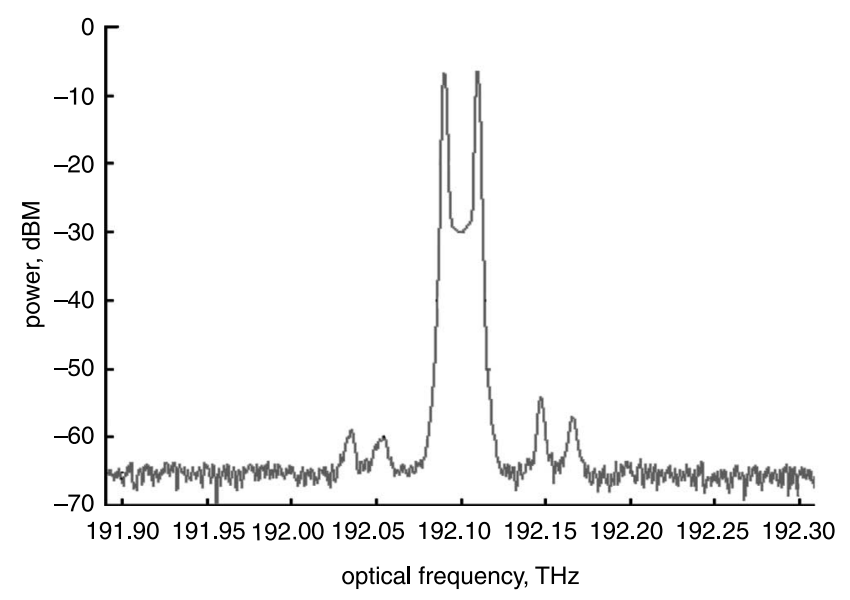

Fig. 7 Time-averaged spectrum of FSK modulation of the DBR laser for ITU channel at $192.1 \mathrm{THz}$

3.2.2 DBR laser results: FSK modulation dynamics of the DBR laser have been investigated in the same way. Figure 7 shows a time-averaged spectrum of FSK modulation with the device at low modulation rates at a sample ITU channel $192.1 \mathrm{THz}$, revealing two optical side tones at $\pm 10 \mathrm{GHz}$ around the central channel frequency. Dynamic side-mode-suppression-ratio (SMSR) is greater than $40 \mathrm{~dB}$, ensuring that the main channel emission prevails against the side channels. However, modulation effects on neighbouring channels $(50 \mathrm{GHz}$ spacing, at $192.05 \mathrm{THz}$ and $192.15 \mathrm{THz}$ ) can be observed. Thus, possible channel interference effects should be considered in the case of a WDM system with channel spacing smaller than $50 \mathrm{GHz}$.

Figure 8 gives an example of TRS measured results, showing the time-resolved spectrum of FSK modulation of the device for a sample ITU channel at $192.1 \mathrm{THz}$. As shown in Fig. 7, FSK deviation of $20 \mathrm{GHz}$ has been achieved with modulation current of $2.2 \mathrm{~mA}$. Here the FSK modulation rate is $78 \mathrm{MHz}(156 \mathrm{Mbit} / \mathrm{s})$.

Figure 9 shows the FM response of the device for the phase current at the bias levels of $3.6 \mathrm{~mA}$. The FM efficiency is plotted against modulation frequency from 1 to $500 \mathrm{MHz}$. It shows a superior performance with a nearly flat response in the low frequency range. Also, it is found that the $3 \mathrm{~dB}$ down cut-off frequency is about $100 \mathrm{MHz}$. This higher FM bandwidth (compared to the GCSR laser) provides a guarantee for FSK labelling rate at $78 \mathrm{Mbit} / \mathrm{s}$ operation, and potential for higher $(125,155 \mathrm{Mbit} / \mathrm{s})$ FSK labelling bite-rate design.

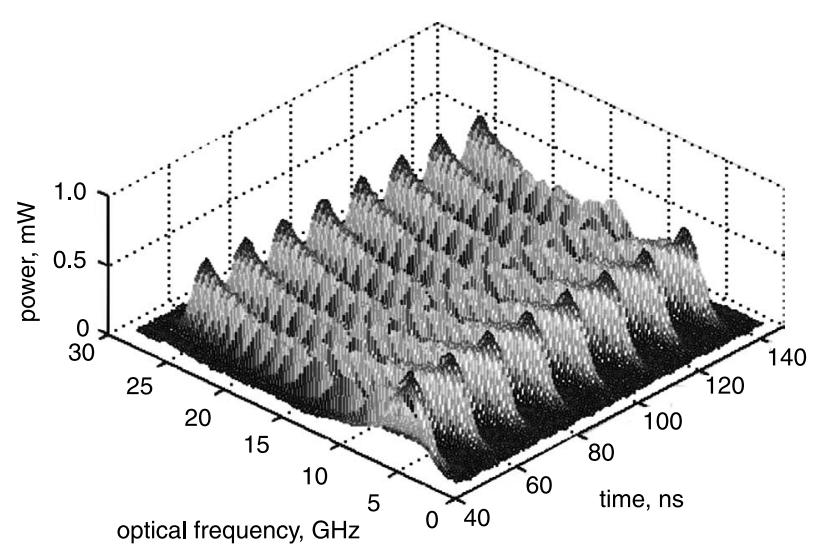

Fig. 8 Time-resolved spectrum of FSK modulation with the DBR laser around ITU channel $192.1 \mathrm{THz}$

166

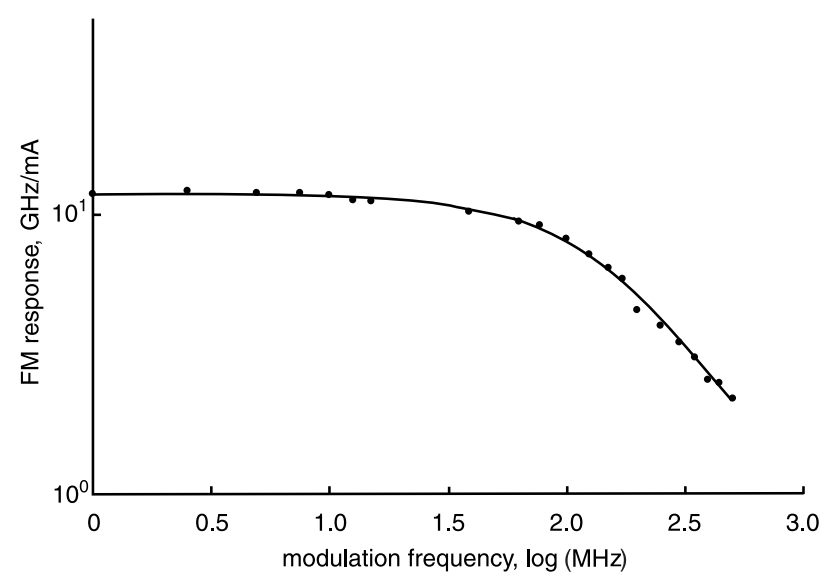

Fig. 9 FM response of the DBR laser with phase section

\section{Comparison of FSK behaviour by grating modulation}

In a further study we focussed on three-section DBR lasers where the reflector grating is tuned instead of the phase section and present theoretical, numerical, and experimental considerations. It is this laser type that is used in the STOLAS demonstrator.

\subsection{Theoretical background}

FSK modulation can be obtained with a tunable laser diode by modulating the tuning current(s), thus causing a modulation of the laser frequency. Preferably only one tuning current has to be changed while the SMSR remains sufficiently large (typically $40 \mathrm{~dB}$ or higher) under the modulation.

Modulation of one of the tuning currents of a tunable laser means that the dynamics of the (FSK) modulation will be determined by the dynamics of the carriers in the (passive) tuning section. These dynamics can be modelled by a simple first order rate equation:

$$
\frac{d N_{r}}{d t}=\frac{\sigma}{e V_{r}} I_{r}-\left(A N_{r}+B N_{r}^{2}+C N_{r}^{3}\right)
$$

where $N_{r}$ is the carrier density, $\sigma$ the current injection efficiency, $V_{r}$ the volume of the reflector section with current $I_{r}$ and $A, B$ and $C$ are the non-radiative, bimolecular and Auger recombination parameters respectively.

The equation above represents a first-order system with a $3 \mathrm{~dB}$ frequency determined by:

$$
f_{3 d B}=\frac{1}{2 \pi}\left(A+2 B N_{r 0}+3 C N_{r 0}^{2}\right)
$$

with $N_{r 0}$ the static carrier density, determined by the bias current through the reflector section.

The numerically calculated values for $f_{3 d B}$ using the following typical parameter values: $A=0.5 \mathrm{E} 8 \mathrm{~s}^{-1}$, $B=100 \mathrm{~s}^{-1} \mu \mathrm{m}^{3}, C=1.3 \times 10^{-5} \mathrm{~s}^{-1} \mu \mathrm{m}^{6}, V_{r}=400 \times 2.5 \times$ $0.1=100 \mu \mathrm{m}^{3}, \sigma=1$, are shown in Table 1. From these values it clearly follows that $f_{3 d B}$ increases with reflector bias current $I_{r \text {-bias }}$ and that FSK modulation at $155 \mathrm{Mbit} / \mathrm{s}$ (corresponding to a modulation frequency of $77.5 \mathrm{MHz}$ ) should be possible.

\subsection{Simulation results}

Simulations were performed using the commercially available software package VPI Componentmaker (www. virtualphotonics.com). 
Table 1: Numerically calculated $3 \mathrm{~dB}$ frequency for FSK modulation against bias reflector current for a threesection DBR laser (FSK modulation obtained by modulating the current through the Bragg reflector tuning section)

\begin{tabular}{lc}
\hline$I_{r-\text { bias }}(\mathrm{mA})$ & $f_{3 d B}(\mathrm{MHz})$ \\
\hline 10 & 98 \\
20 & 149 \\
30 & 190 \\
35 & 209 \\
40 & 227 \\
45 & 244
\end{tabular}

The FSK small signal response was obtained by converting frequency variations into power variations using a (tunable) optical filter and by calculating the RF-spectrum of those power variations. Figure 10 shows the simulation results for a three-section DBR laser with an output power $P_{\text {out }}=27.5 \mathrm{~mW}$ and for a reflector bias current $I_{r \text {-bias }}=45 \mathrm{~mA}$ and a modulation current amplitude $I_{m}=3 \mathrm{~mA}$ (corresponding to a frequency swing $\Delta f=11 \mathrm{GHz})$.

The $3 \mathrm{~dB}$ frequency can be obtained from this graph by fitting the simulated values to the first order model from the previous paragraph, the fitted values are also shown in Fig. 10. We determined $f_{3 d B}$ for three different bias currents through the reflector section. For current values of 23, 33.5 and $45 \mathrm{~mA}$ we obtained $3 \mathrm{~dB}$ frequencies of 165, 208 and $254 \mathrm{MHz}$ respectively. Again we see that $f_{3 d B}$ increases with reflector bias current $I_{r \text {-bias }}$ and that FSK modulation at $155 \mathrm{Mbit} / \mathrm{s}$ should be possible.

When we compare these values of $f_{3 d B}$ with those in Table 1, we see good agreement between the simulation and the theoretical results. The values for $f_{3 d B}$ are slightly different because the parameters values used in the simulations were not identical to those used in the theoretical calculations.

\subsection{Experimental results}

Finally, the theoretical and simulation results are also confirmed by experimental results. Figure 11 shows the experimentally obtained $3 \mathrm{~dB}$ frequencies for FSK modulation of a three-section DBR laser, again for different bias reflector currents. Again it follows that $f_{3 d B}$ increases for increasing $I_{r \text {-bias }}$ and that FSK modulation at $155 \mathrm{Mbit} / \mathrm{s}$

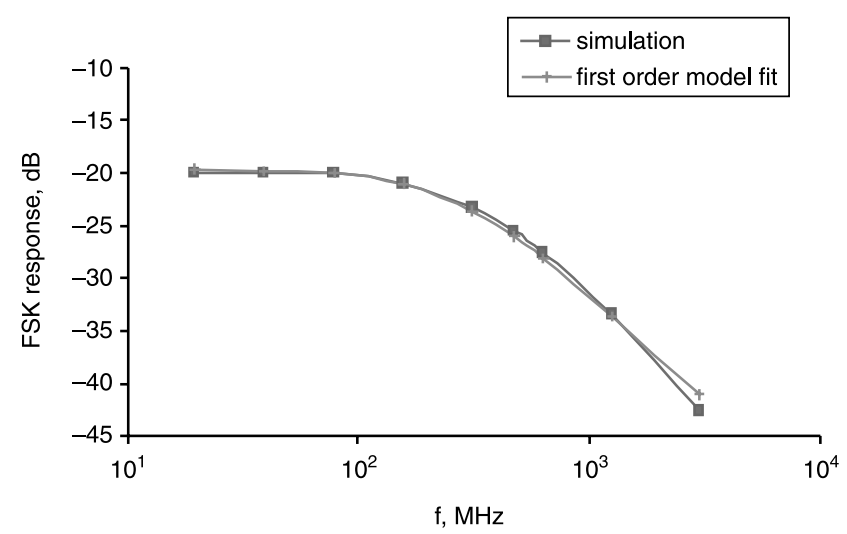

Fig. 10 Simulated FSK small signal modulation response of a three-section DBR laser $\left(P_{\text {out }}=27.5 \mathrm{~mW}, \quad I_{r \text {-bias }}=45 \mathrm{~mA}\right.$, $I_{m}=3 \mathrm{~mA}$ ) and theoretical fit to the first order model

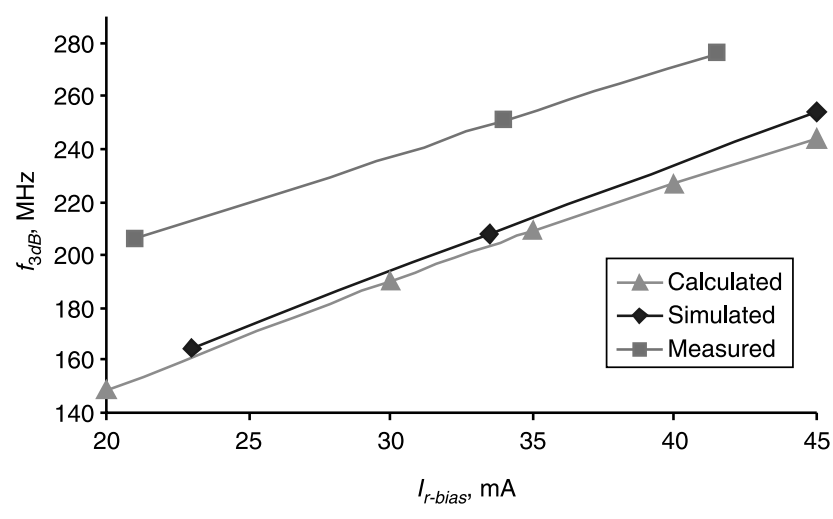

Fig. 11 Experimental, simulated and theoretical values for $f_{3 d B}$ of a three-section DBR laser against bias reflector current $I_{r \text {-bias }}$

should be possible. The experimentally obtained values for $f_{3 d B}$ are higher than the theoretical ones owing to the different values of $A, B, C$ and $V_{r}$ for the lasers used in the experiments (these values are unknown to us).

Extrapolation of the experimental results to zero $I_{r \text {-bias }}$ indicates that a $3 \mathrm{~dB}$ bandwidth of over $100 \mathrm{MHz}$ can even be obtained for zero bias current through the reflector section.

Large signal FSK modulation experiments were also performed. Figure 12 shows a typical eye diagram for 155 Mbit/s FSK modulation. The bias current through the reflector section was $21 \mathrm{~mA}$, the modulation current was $3.6 \mathrm{~mA}$ and the frequency swing was $17 \mathrm{GHz}$. The eye is clearly open, as was to be expected from the $f_{3 d B}$ values in Fig. 12. The maximum obtainable frequency swing (required to be $20 \mathrm{GHz}$ ) is determined by the width of the frequency plateaus in the tuning table of the laser. This width typically increases with increasing reflector current. For the lasers used in the experiments the maximum obtainable frequency swing was a bit less than $20 \mathrm{GHz}$. For the lasers to be used in the STOLAS demonstrator, a frequency swing of $20 \mathrm{GHz}$ would be readily obtainable.

It has been shown theoretically, numerically and experimentally that $155 \mathrm{Mbit} / \mathrm{s}$ FSK modulation using three-section tunable lasers is possible.

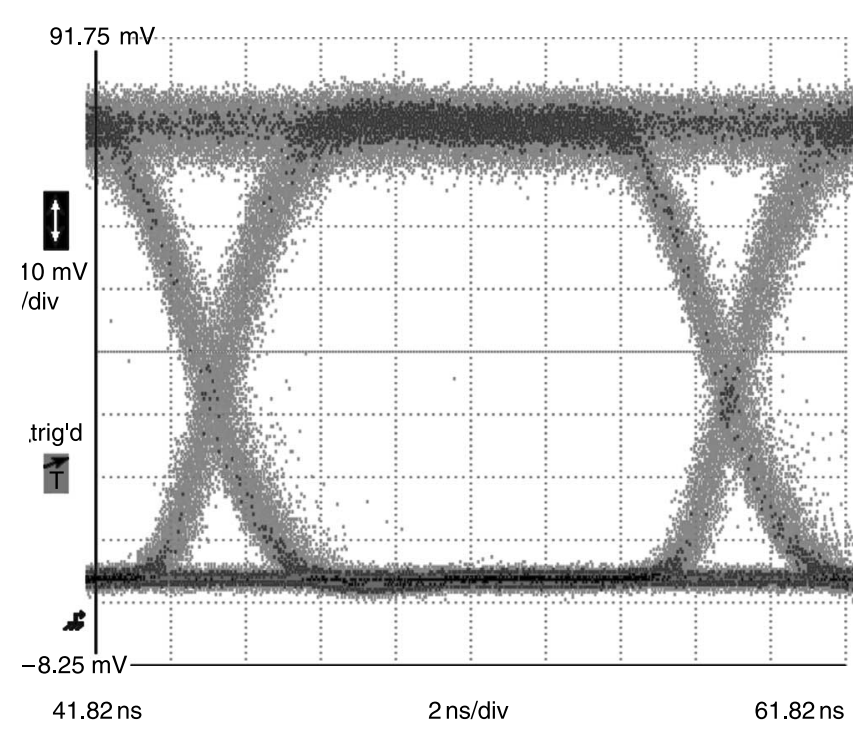

Fig. 12 Experimentally obtained eye diagram for FSK modulation of a three-section $D B R$ laser for $I_{r-b i a s}=21 \mathrm{~mA}$ and $I_{m}=3.6 \mathrm{~mA}$ 


\section{Thermal effects in wavelength switching and FSK}

In this Section we describe the implications of thermal effects on wavelength switching. For multi-section tunable lasers, the wavelength switching dynamics are determined by carrier density and temperature, both resulting from the variation of the tuning section current(s). After a fast wavelength switching has taken place, a thermal transient induced by the tuning current(s) swing causes a slow wavelength drift towards a steady state value. The thermal behaviour plays no significant role on the high speed switching characteristics; however it broadens the spectrum of the optical signal and degrades the switching time at lower data rates. Importantly, while it sets an upper limit to wavelength-channel switching rate, the FSK label will not be degraded by thermals as long as the labelling rate is well above the thermal transients ability to keep up. This was an original feature of the STOLAS concept.

Frequency modulation (FM) response of the GCSR laser is investigated theoretically and is compared to measurement [8]. A red-shifted thermal contribution that results in a dip in the overall FM response, at the low frequency range, is observed. Measured thermal transients of wavelength switching shows that settling time is around 300-500 ns, indicating a guard time at least of $500 \mathrm{~ns}$ for timing control design.

\subsection{FM response}

The FM response stems mainly from two opposite contributions; the carrier and the thermal response. The thermal response is exhibited at low frequencies, while the carrier response is flat at the lower frequencies and has a resonance-like behaviour at higher frequencies. Both of these responses are analysed separately and under small signal approximation they can be added together. The full FM response written in terms of the Laplace transform [9] is given by:

$$
H(s)=H_{c}(s)+H_{t}(s)
$$

where $H_{c}(s)$ and $H_{t}(s)$ denote the carrier FM response and the thermal FM contributions respectively.

By solving heat diffusion equations in the chip and the sub-mount, we can evaluate the transfer function of the thermal FM response. To model the thermal effect, the laser is modelled as an equivalent single layer and the heat flow is assumed to be one-dimensional. The heat source is located at the centre of the chip. The conduction equation for onedimensional heat flow [10] is expressed as:

$$
\frac{\partial^{2}[T(x ; t)]}{\partial x^{2}}-\frac{1}{\kappa} \frac{\partial[T(x ; t)]}{\partial t}=0
$$

The heat is generated by two mechanisms, ohmic and optical loss, both of which are related to inputs currents. We solve this equation by taking its Laplace transformation and solve that using the various boundary and initial conditions. The solution then is the response of the temperature $T(s)$ averaged over the laser chip:

$$
T(s)=\frac{K_{R} Q(s)}{2 q l}\left[\tanh (q l)+\tanh \left(\frac{q l}{2}\right)\right]
$$

Here $Q(s)$ is the Laplace transformation of the heat generated in the chip owing to the injection current, $K_{R}$ is the thermal resistance, $q=\sqrt{s / \kappa}, \kappa$ is thermal diffusivity, $l$ is effective half-thickness being the maximum $x$, and $s$ is the Laplace variable conjugated to time $t$.

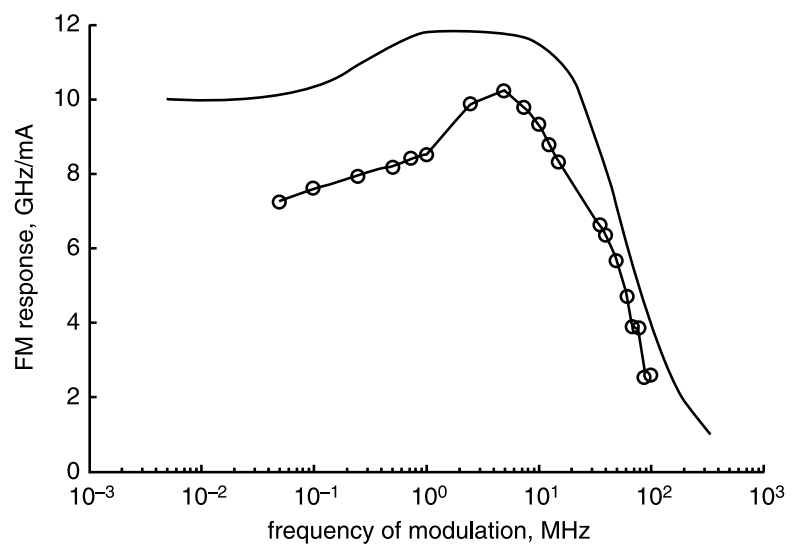

Fig. 13 Simulated FM response of the GCSR laser, compared with measured results

At the lower frequencies the carrier response can be considered constant and flat. The cut-off frequency is determined by the carrier lifetime in the passive section. By combining the carrier and the thermal effects, a simulated result of the full FM response of the GCSR laser is presented in Fig. 13, compared with actual measurements from the laser [8]. It is clear that there is a dip in the FM response at low frequency range $(<3 \mathrm{MHz})$. This is as a result of the addition of a blue-shifted carrier contribution and a red-shifted thermal contribution.

\subsection{Thermal transients}

Thermal effects on wavelength switching dynamics of the device are measured by the time-resolved spectral (TRS) technique [8]. Figure 14 gives examples of measured results. The time variation of optical frequency in response to the switching signals can be seen clearly from this Figure.
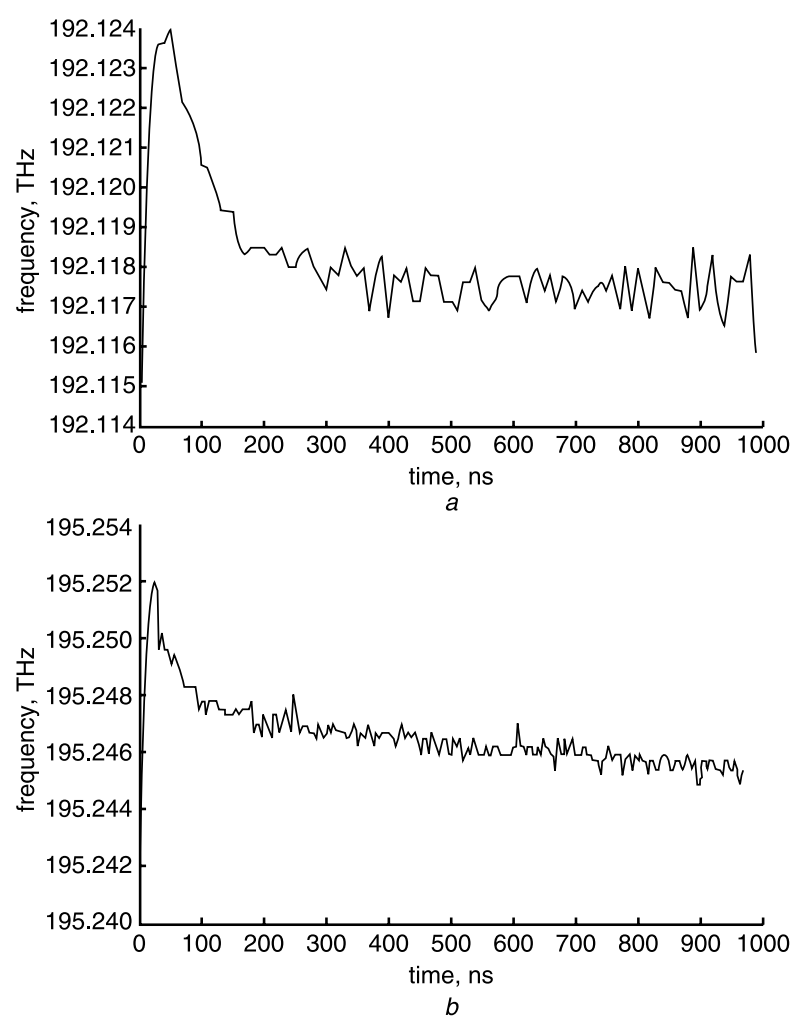

Fig. 14 Resolved plots from TRS measurements, showing frequency evolution with time owing to thermal effects

$a$ Switching current of $2.5 \mathrm{~mA}$ into phase section

$b$ Switching current of $8 \mathrm{~mA}$ into coupler section 
When the switching event occurs, the laser frequency jumps rapidly owing to the carrier effect and then slowly drifts toward a steady state value owing to the thermal effect.

From Fig. 14, it can be found that frequency excursions in first few ten nanoseconds is about $6-7 \mathrm{GHz}$. However, the laser settles down to within $2 \mathrm{GHz}$ after $300 \mathrm{~ns}$. Similar results have been obtained under various test conditions. It has been shown that the settling time of the first time constant for thermal drift is $300-500 \mathrm{~ns}$. Clearly, a guard time at least of $500 \mathrm{~ns}$ is need for avoidance of thermal transients and so that the laser can switch from one wavelength to another wavelength within the accuracy of $\pm 5 \mathrm{GHz}$ as required

\section{Conclusion}

Implementation, performance and behaviour of FSK modulation with various tunable lasers have been investigated. FSK modulation with $\pm 10 \mathrm{GHz}$ tones by using one tuning section only, either phase or grating, has been demonstrated. Also it was found that dynamic side-mode-suppression-ratio under FSK modulation remains $35-40 \mathrm{~dB}$. Reasonable labelling rates $(78 \mathrm{Mbit} / \mathrm{s})$ can be achieved with the devices. Experimental results in addition to theoretical results from three-section DBR lasers also indicate their potential for higher labelling rates $(125,155 \mathrm{Mbit} / \mathrm{s})$.

Thermal effects on wavelength switching dynamics of tunable devices have been also studied. A red-shifted thermal contribution that results in a dip in the overall FM response, at the low frequency range has been observed. Degradation to switching time and control precision of wavelength have been measured. It has been shown that a guard time of at least $500 \mathrm{~ns}$ is needed when the laser switches between wavelength channels, in order to avoid thermal transients and ensure laser operation at ITU channels within the accuracy of $\pm 5 \mathrm{GHz}$ as required.

\section{Acknowledgments}

DBR lasers were from Bookham Technology (UK); GCSR lasers from ADC (USA). The STOLAS project is funded in the EU-IST programme 2001-2004. Support from Eircom PLC Ireland and PXIT, Dublin, Ireland/PXIT Inc. (USA) is gratefully acknowledged.

\section{References}

1 Koonen, T., Morthier, G., Jennen, J., de Waardt, H., and Demeester, P. 'Optical packet routing in IP-over-WDM networks deploying two-level optical labelling', ECOC, Amsterdam, The Netherlands, 30 Sept.-4 Oct. 2001, paper Th.L.2.1, pp. 608-609

2 Vlachos, K., Jennen, J., Monroy, I.T., and Sulur.: 'The orthogonal coding scheme for implementation of the optical label stack', STOLAS Deliverable D3.1.1, August 2002

3 O'Dowd, R., O'Duill, S., Mulvihill, G., O'Gorman, N., and Yu, Y. 'Frequency plan and wavelength switching limits for widely tunable semiconductor transmitters', IEEE J. Sel. Top. Quantum Electron. 2001, 7, (2), pp. 259-269

4 Yu, Y., O'Duill, S., Mulvihill, G., O’Dowd, R., Moeyersoon, B., and Morthier, G.: 'Wavelength switching possibilities of tunable laser diodes', STOLAS Deliverable D2.1.2, Nov. 2002

5 Vlachos, K., Zheng, J., Cheyns, J., Sulur, Chi, N., Van Breusegem, E. Tafur Monroy, I., Jennen, J.G.L., Holm-Nielsen, P.V., Peucheret, C., O'Dowd, R., Demeemster, P., and Koonen, A.M.J.: 'An optical IM/FSK coding technique for the implementation of a label-controlled arrayed waveguide packet router', J. Lightwave Technol., 2003, 21, pp. 2617-2628

6 Yu, Y., Mulvihill, G., O'Duill, S., and O'Dowd, R.: 'Performance implications of wide-band lasers for FSK modulation labeling scheme', IEEE Photon. Technol. Lett., 2004, 16, pp. 39-41

7 Chi, N., Zhang, J., Holm-Nielsen, P.V., Kozicki, B., Peucheret, C. Monroy, I.T., and O'Duill, S.: 'Experimental results on single-channel transmission of orthogonally modulated signals', STOLAS Deliverable D4.2.1, July 2003

8 Mulvihill, G., Yu, Y., O'Duill, S., and O'Dowd, R.: 'Thermal contribution to wavelength switching characteristics of widely tunable lasers'. LEOS, Tucson, Arizona, 26-30 Oct. 2003, paper WT4

9 Roudas, I., Jaouen, Y., Prado, J., Vallet, R., and Gallion, P.: 'Recursive simulation models of the semiconductor laser modulation characteristics for accurate performance evaluation of coherent optical CPFSK systems', J. Lightwave Technol., 1995, 13, pp. 2258-2269

10 Carlsaw, H.S., and Jaeger, J.C.: 'Conduction of heat in solids' (Oxford University Press, London, 1959)

11 Correc, P., Girard, O., and Faria, Jr., I.F.d.: 'On the thermal contibution to the FM response of DFB lasers: theory and experiment', IEEE J. Quantum Electron, 1994, 30, pp. 2485-2489 